\title{
LA REVISTA DE HISTORIA DE AMÉRICA COMO LABORATORIO DE PRÁCTICAS
}

\author{
Alexandra Pita González \\ Universidad de Colima
}

\begin{abstract}
1 festejarse la publicación de los 200 números de la revista A Historia Mexicana en 2001, se elaboró un número especial dedicado a otras revistas de historia publicadas en México. A juicio de Javier Garciadiego, tres publicaciones estuvieron ausentes: la Revista de Historia de América, Cuadernos Americanos y Mexican Studies. La primera, fundada en 1938 por Silvio Zavala, era importante porque a pesar de que trataba de un ámbito continental, sirvió "como único laboratorio en el que se formaron, redactando notas y reseñas, varios de los jóvenes historiadores mexicanos de entonces". ${ }^{1}$

En efecto, como se señala en las siguientes páginas, la Revista de Historia de América (RHA) fue un antecedente importante no sólo porque en ella participaron algunos maestros y numerosos estudiantes del Centro de Estudios Históricos (CEH) de El Colegio de México. En ella se practicó de manera sistemática el ejercicio de la lectura y la crítica de documentos, para formar historiadores vinculados a la investigación. Sentó las bases de los elementos que debía componer una revista
\end{abstract}

1 Garciadiego, “Revistas revisitadas” (202), 2001, pp. 222-223. 
académica de y para profesionales: una publicación que formara al lector especializado por medio de una alta dosis de documentos para sustentar las ideas; un enorme repositorio de libros y artículos de historia para leer y criticar; un espacio donde recordar y homenajear a los historiadores fallecidos; $y$, por último, un centro de noticias sobre lo que acontecía en el ámbito institucional en el continente. Así, para 1951, cuando se fundó Historia Mexicana, la publicación era un modelo a seguir o superar de lo que debía ser una revista dedicada a profesionalizar la historia.

Cabe aclarar que, dada la extensión de este trabajo y a que el análisis de esta publicación es objeto de un estudio mayor, ${ }^{2}$ nos limitaremos a señalar en los primeros dos apartados algunos aspectos de la publicación que permiten delinear sus rasgos más importantes, para a continuación puntualizar la participación de maestros y alumnos del CEH.

\section{UNA REVISTA CIENTÍFICA Y AMERICANA}

En 1937, los historiadores Silvio Zavala y Lewis Hanke visitaron al geógrafo Pedro Sánchez, director del Instituto Panamericano de Geografía e Historia (IPGH), para proponerle fundar una publicación de historia que tuviera alcance continental. La reunión dio muy buenos resultados y al año siguiente apareció el primer número de la revista. Para comprender su origen es necesario enunciar tres aspectos significativos de su contexto.

2 Junto a María del Carmen Grillo estudiamos los primeros diez años de vida de la publicación, proyecto financiado por el IPGH. Como resultado se realizó un artículo para señalar las estrategias metodológicas y un libro (en prensa) en el que se analiza al detalle la publicación desde una perspectiva que privilegia el lugar de Zavala como editor, pero que muestra la red editorial y académica que se conformó. Véase Pita, Grillo y Morales, "La datificación como propuesta de análisis", y PITA y Grillo, La Revista de Historia de América. Silvio Zavala como editor y la formación de redes académicas, 1938-1948. 
De inicio, la profesionalización de la historia en México, la cual tuvo su mayor auge en las décadas de 1930 y $1940 .{ }^{3}$ Este proceso se asocia a la fundación de instituciones académicas: Instituto de Investigaciones Estéticas de la UnAM (1935), La Casa de España (1938), el Instituto Nacional de Antropología e Historia (1939) y El Colegio de México (1940). ${ }^{4}$ Se vincula también con la aparición de revistas académicas, como un medio asociado a la sistematización de la enseñanza de la historia como profesión, lo cual a su vez aumentaba la producción académica de los historiadores. ${ }^{5}$

Al decir de Luis González, durante esta "fiebre de las fundaciones" se crearon "albergues de la cultura humanística y particularmente cliomática”. El signo de cambio impactó también en el mundo editorial. Se crearon Porrúa, Fondo de Cultura Económica (con su publicación El Trimestre Económico), Jus y la imprenta de la UnAM. Aparecen publicaciones periódicas especializadas: Boletín del Archivo General de la Nación (1930), Ábside (1937), Divulgaciones Históricas (1939), Revista de la Facultad de Filosofía y Letras (1941), Cuadernos

${ }^{3}$ Para que este cambio se produjera, se conjugaron factores políticos internos y externos (la consolidación del régimen revolucionario durante el gobierno de Cárdenas, el impacto de la Guerra Civil española), así como la circulación de un "lenguaje histórico de corte nacionalista liberal" donde se encuentran ya elementos que caracterizarán el discurso de la historia como ciencia-heredera de la búsqueda por alcanzar una verdad "imparcial y objetiva, expuesta por el historiador alemán Leopoldo von Ranke-. ZermeÑo, "La historiografía en México" (248), (2013), pp. 1695-1697.

${ }^{4}$ Para Moctezuma Franco, puede observarse la profesionalización de la historia en México cuando se "cran instituciones dedicadas expresamente para formar historiadores". Moctezuma Franco, "El camino de la historia hacia su institucionalización”, p. 47. En cambio, para Alejandra Pinal, en 1938 no se institucionalizó la historia, sino que se oficializó como profesión, por lo que es necesario entender que el proceso inicia desde el siglo xix. Pinal, Vivir para historiar, historiar para vivir, pp. 178-179, 193, 196.

5 Jiménez, “50 años de Historia Mexicana” (3), 1952, pp. 451-452. 
Americanos (1941), Anuario de Historia y Revista de Historia de América (1938). ${ }^{6}$

Esto se asocia al segundo aspecto, que fue la consolidación profesional del propio Zavala, quien para fines de la década de 1930 era ya un historiador reconocido. Ha sido señalada la importancia que tuvieron sus años formativos en España bajo la tutela de Rafael Altamira en la Universidad Central de Madrid y en el Centro de Estudios Históricos, fundado al calor de la Junta de Ampliación de Estudios desde principios de siglo, donde se reunieron muchos de los más destacados historiadores, literatos y filólogos de España, al igual que en la Residencia de Estudiantes.

Menos importancia se le ha dado a su participación en la revista Tierra Firme, ${ }^{7}$ pero significaría una toma de conciencia de la necesidad de crear espacios de circulación de ideas americanistas para un público interesado en obtener información precisa. En referencia al americanismo, era el lazo que unió a los miembros de la redacción de la revista española, entre los cuales se encontraba Zavala. ${ }^{8}$ Como recordaría Antonio Saborit, el levantamiento militar en España, en el verano de 1936, explica el regreso a México de Zavala, pero también el que, al hacerlo, sus primeros pasos hubieran sido incorporarse al ámbito de la

6 González, “Historia de la historia” (58-59), 1965-1966, pp. 197-199 y GonzÁlez, “La pasión del nido” (100), 1976, p. 531.

7 Tierra Firme fue una publicación trimestral que apareció entre los años 1935 y 1937. Dependía de la Sección Hispanoamericana del Centro de Estudios Históricos. Fue impulsado por Américo Castro y dirigida por Enrique Díez Canedo. Promovió un "americanismo liberal” que buscaba definir la identidad española a través de la relación con Hispanoamérica. La revista publicó 8 números, pero cesó a consecuencia de la Guerra Civil pues buena parte de sus miembros se exiliaron. MorA, "El impulso renovador del americanismo durante la Segunda República”, pp. 10-11.

8 Bajo la batuta de Américo Castro se agrupó un pequeño grupo de redacción conformado por jóvenes estudiantes: Silvio Zavala, Ángel Rosenblat, Rodolfo Barón Castro, Ramón Iglesia, Raquel Lesteiro. En la revista Zavala publicó durante un breve periodo de dos años 14 artículos. Bernabéu y Naranjo, “Historia contra la 'desmemoria' y el olvido”, pp. 98, 164-165. 
investigación histórica y "empeñarse en crear el espacio editorial especializado" que fue la Revista de Historia de América.

Entender por qué Zavala buscó la ayuda del IPGH nos remite a otra dimensión del contexto. El organismo regional, dependiente de la Unión Panamericana, cuya sede estaba en la ciudad de México desde su instalación en 1930, buscó contribuir al avance científico de las disciplinas de la geografía y la historia y acercar a los países miembro mediante estos campos académicos bajo el cobijo de la neutralidad científica. De manera indirecta, esta meta ayudaría a limar asperezas con aquellos intelectuales latinoamericanos que veían con recelo -cuando no con francas críticas-al panamericanismo. Además, al ser una publicación del Instituto permitía abrir la posibilidad de contribuciones de todo el continente en varios idiomas. Permitía también centrarse en una historia compartida (enfocada sobre todo en la época colonial, pero también en los primeros años de la vida independiente), desde una mirada que superara los estrechos límites nacionales. Como se expresaba en el primer número, el conocimiento de los problemas comunes a todos los países del continente ofrece una ventaja al permitir "escribir con mayor acierto las historias nacionales" ${ }^{10}$ Erika Pani apunta que el llamado de Zavala a través de la $R H A$ “ofrecía menos un programa que un espacio para la historia continental" y lo hacía desde un vínculo institucional que le otorgaba "vuelo político y arraigo burocrático". ${ }^{11}$

\section{UNA RED DE HISTORIADORES, ARCHIVISTAS Y BIBLIOTECARIOS}

El proyecto de Zavala funcionó durante largo tiempo con sus altibajos y cambios coyunturales. La publicación trascendió los límites nacionales para crear una red de colaboradores

\footnotetext{
9 SAborit, "Silvio Zavala: en su homenaje" (251), 2014, p. 1423.

10 RHA, “Propósitos”, 1 (mar. 1938), pp. v y vi.

11 PANi, "Silvio Zavala y la historia de América”, pp. 178-179.
} 
especialistas que presentaran trabajos de "carácter científico" y no de divulgación. Dado el valor que se le otorgaba al documento, no resultó extraño que esta red estuviera compuesta por historiadores, archivistas y bibliotecarios (o historiadores versátiles que tuvieran dominio de lo que entonces se conocía como ciencias auxiliares).

Esta combinación era fundamental y congruente con el perfil cientificista de la revista, ${ }^{12}$ la cual se compuso de diversas secciones durante sus primeros años: artículos, reseñas, revistas, notas bibliográficas, noticias y notas necrológicas. ${ }^{13}$ La primera, aunque no ocupó muchas páginas, da muestra de la preocupación por las fuentes documentales, los centros que las resguardan y la visión desde la cual se podían interpretar. La sección dedicada a rendir homenaje a los historiadores fallecidos es significativa al trazar vínculos académicos, pero porcentualmente ocupa un lugar menor. En cambio, las tres secciones dedicadas a comentar las novedades en la producción académica (revistas, notas bibliográficas y reseñas) ocuparon la mayoría de las páginas de cada número. Por su parte, la sección dedicada a las noticias se deslindaba de lo académico para adentrarse en lo institucional.

Aunque la red era representativa de su enfoque internacional, al contar con colaboradores de casi todos los países americanos, se concentró en México, Argentina y Estados Unidos. En el primer país se encontraba la sede del IPGH y el director Zavala, sobre quien recaía la responsabilidad de planear, ejecutar y corregir tanto los aspectos de forma como los de contenido de

12 Alcancía, Letras de México, Cuadernos Americanos y Filosofía y Letras, entre otras, representaban la corriente historicista, mientras que la Revista de Historia de América representaría a la corriente cientificista que defendía "el trabajo de archivo y el apego a los hechos en la búsqueda de la verdad histórica”. Moctezuma Franco, "El camino de la historia hacia su institucionalización”, pp. 50-51, 58.

13 Con ciertos cambios: revistas fue incorporada a notas bibliográficas en 1941; noticias aparece en 1945. 
la publicación. Para ello escribió numerosas cartas a posibles colaboradores, leyó y seleccionó el material, y envió a los autores sugerencias y cambios. Le irritaban los errores, por lo que fue un lector acucioso para detectarlos o enmendarlos con fe de erratas. Estuvo atento a cada detalle del contenido de la publicación, así como de los aspectos técnicos (imprentas, tipo de papel, tamaño de letras, tinta, tiraje, costos). A esta labor sumó otras para asegurar que autores, temas y publicaciones fueran representativas del continente. Junto con Pedro Sánchez (director del IPGH), seleccionó al equipo editorial y a los miembros del Consejo Directivo, a quienes distribuyó la tarea de conseguir buenos trabajos en sus países, al tiempo que solicitó difundieran los números publicados.

Para ello, conformó un equipo editorial, compuesto por mexicanos y extranjeros radicados por distintos motivos en la ciudad de México: el hondureño Rafael Heliodoro Valle; los españoles Felipe Teixedor, Agustín Millares Carlo y Javier Malagón; los mexicanos José Ignacio Rubio Mañé, Francisco Monterde, Ernesto de la Torre Villar y Susana Uribe. Aunque parezca un grupo relativamente numeroso, no lo era porque no todos participaron en la misma época. En los primeros tres años (1938-1940) Zavala contaba oficialmente con Teixedor (1938 y 1939), pero en la práctica sólo con Valle, Monterde y Rubio Mañé. El hondureño era parte del Consejo Directivo, no del Comité Editorial, por lo que su labor se restringía a coordinar y elaborar la mayor parte de las notas bibliográficas. Discrepancias sobre la forma de normalizar las notas, así como retrasos y errores, hicieron que abandonara esta tarea, aunque se mantuvo cercano a la publicación y al Instituto. Los dos mexicanos se encargaban de varias labores: redactar la totalidad de notas para la sección Revistas, ayudar a Zavala a corregir, comunicarse con los autores, vigilar el proceso editorial desde el manuscrito hasta las pruebas de imprenta y sobretiros y hacerles llegar los pagos correspondientes. Otra de las tareas no menores consistía 
en vigilar con las imprentas la edición para asegurar así la periodicidad y el tiraje. Aunque compartían estas labores con Zavala, recaían en ellos (y fundamentalmente en Rubio Mañé) cuando el director salía en sus viajes de estudios.

Pese a las calamidades de la segunda guerra mundial, este pequeño grupo siguió trabajando desde 1941 hasta 1945 con el reemplazo de Valle, Agustín Millares Carlo, quien -como mencionaremos más adelante-, fue un integrante estratégico para el plan trazado, aunque no aparece como miembro del equipo editorial. En 1946 hubo cierta turbulencia para la revista, pues tras la creación de la Comisión de Historia (presidida por Zavala), la publicación tuvo que realizar algunos ajustes para asegurar su permanencia. A pesar de ello, sus números continuaron saliendo con la periodicidad adecuada y el pequeño equipo (Zavala, Monterde y Rubio Mañé) siguió a cargo. En 1948 hubo un reacomodo importante pues, aunque Zavala sigue apareciendo como director, se suma Javier Malagón, quien aparece como secretario del editor, mientras como redactores se agregaron los nombres de Agustín Millares Carlo, Ignacio Rubio Mañé, Ernesto de la Torre Villar y Susana Uribe. Todos ellos aparecen de manera conjunta como los "Editores”. Esto no sólo significó la creación de cargos, sino una nueva distribución del trabajo.

El papel protagónico de la revista americanista, editada en México, contribuyó a moderar el predominio intelectual de Argentina y Estados Unidos en este campo pues, como comunicó Zavala a Alfonso Reyes, "las bases de la investigación moderna hispanoamericana” descansaban en estos dos países. Reyes entendió bien su argumento al responderles "hace Usted bien en desear que reforcemos un poco en México las investigaciones hispanoamericanas para ponerlas a la altura de otros países del continente". ${ }^{14}$

${ }_{14}$ Carta de Alfonso Reyes a Silvio Zavala, 26 de abril de 1939, en EnRíquez, Fronteras conquistadas, p. 42. 
En todo caso, no es extraño encontrar las colaboraciones desde Argentina de José Torre Revello y Sara Sabor Vila, quienes aportaron sus esfuerzos en la elaboración de reseñas y notas bibliográficas de la producción de Argentina, Uruguay y Paraguay. La participación de Torre es de destacar porque en la numerosa correspondencia que entabló con Zavala se puede observar el trabajo minucioso de selección, ordenamiento y clasificación del material. De hecho, Zavala lo puso como modelo a seguir para todos los colaboradores de reseñas y notas, al señalar en repetidas ocasiones su prolijidad y mesura entre la descripción y el análisis. También debe mencionarse a otro destacado intelectual argentino, Ricardo Levene, quien publicó algunos artículos, pero sobre todo sirvió de enlace con la comunidad de historiadores argentinos.

En el caso de Estados Unidos, el vínculo más fuerte fue con Lewis Hanke, quien apareció en pocas ocasiones como autor, pero tuvo una función relevante como mediador de redes entre la revista y la academia estadounidense. En virtud de ello, Hanke se encargó no sólo de lograr que las editoriales y autores enviaran sus libros y revistas para reseñarse (o al menos lograr una nota), sino de conseguir entre sus compatriotas material para publicar en todas las secciones de la revista, como fue el caso de Bert James Lowenberg, quien se encargó de redactar numerosas notas bibliográficas y reseñas de libros editados en su país. En tanto Hanke fue director de la División de Asuntos Hispánicos de la Biblioteca del Congreso, tuvo una posición privilegiada para obtener materiales valiosos, los cuales nutrieron a la revista.

\section{LA PARTICIPACIÓN DE MAESTROS Y ESTUDIANTES}

En 1941, Zavala se convirtió en el director del Centro de Estudios Históricos (CEH) de El Colegio de México y pidió a Reyes que le permitiera mantener su responsabilidad como director 
de la $R H A$ porque coincidía con la propuesta académica. ${ }^{15} \mathrm{Al}$ mismo tiempo que dictaba sus seminarios, investigaba y escribía libros, dirigía el Centro y también la revista (a lo que sumó poco después su participación en la Comisión de Historia del IPGH y en el Museo Nacional).

No es extraño que dada su labor silenciosa como director, como autor apareció en pocos artículos de su revista, un número mayor de reseñas y ayudó una innumerable cantidad de veces a completar la minuciosa labor de las notas bibliográficas y noticias. Al respecto, otros profesores del CEH de El Colegio de México también participaron en la publicación: Arturo Arnaiz y Freg, François Chevalier, Juan Iguíñiz, José María Miquel i Vergés y José Miranda. A éstos hay que agregar la colaboración de Daniel Cosío Villegas y de Alfonso Reyes. Si bien estas contribuciones fueron escasas, publicando un par de artículos y reseñas cada uno, representaban una importante presencia intelectual, el sello de una ya prestigiosa institución. En cambio, otro grupo de profesores participó gran cantidad de veces, convirtiéndose en colaboradores asiduos: Rafael Altamira, Agustín Millares Carlo, José Rojas Garcidueñas y Bert James Lowenberg.

Rafael Altamira, su maestro, de quien Zavala aprendió tanto durante su estancia en Madrid y a quien ayudó a trasladarse a este continente, a raíz de la Guerra Civil española, fue un caso especial. No sólo fue el autor con mayor cantidad de artículos publicados (también participó en reseñas y notas necrológicas), sino que se convirtió en un referente de la publicación por la cantidad de veces que fue citado. En el caso de Agustín Millares Carlo, su labor fue fundamental pues reemplazó a Rafael Heliodoro Valle como coordinador de la sección de notas bibliográficas desde 1941, introduciendo una serie de cambios

15 Véase la correspondencia cruzada entre Reyes y Zavala en EnRíquez, Fronteras conquistadas, pp. 93-95. 
en la sección para sistematizar la labor. Se hizo cargo de la organización del archivo y la biblioteca del IPGH (para ordenar el material que era enviado a la redacción), escribió numerosas notas y reseñas, así como se ocupó de la elaboración de fichas que posteriormente servirían para el índice anual. Para este trabajo metódico contó con la ayuda de José Rojas Garcidueñas, quien además se responsabilizó de redactar una parte considerable de las reseñas. ${ }^{16}$

El panorama es diferente al observar a los estudiantes del $\mathrm{CEH}$, quienes participaron de manera activa en la revista. Recordemos que por la propia dinámica de los seminarios que impartían, los estudiantes tenían que realizar trabajos individuales y colectivos bajo la conducción de un profesor. Además, el propósito del plan de estudios era formar "historiadores de América", los que debían tener la capacidad de "enseñar e investigar la historia de nuestro continente". ${ }^{17}$ No por casualidad, Luis González recuerda que Zavala, como director, aplicaba "una pedagogía de invernadero y la búsqueda ratonera", donde los alumnos debían de manera simultánea "asistir a congresos

16 Su caso es interesante. No era miembro formal del comité de edición, pero se convirtió en un colaborador permanente al ayudar en la labor que realizaba José Ignacio Rubio Mañé de generar notas en la sección dedicada a revistas sobre los artículos que les parecían más interesantes. Esta tarea recaía en Rubio Mañé y en Monterde, quien también se encargaba de la corrección de estilo, por lo que participó poco en este quehacer. A medida que aumentaba la cantidad de revistas que enviaban a la redacción, Zavala decidió que Arnaíz y Rojas le ayudaran. Como esta sección desapareció en 1941 (al ser incorporada a las notas bibliográficas), Rojas se dedicó de lleno a la sección reseñas (en la que ya colaboraba desde antes), convirtiéndose en uno de los principales reseñistas de la revista sobre obras escritas en español a partir de su incorporación en el número 8 (1939). Siguió colaborando también en la sección de notas bibliográficas.

${ }_{17}$ Algunos de estos trabajos eran publicados en obras colectivas con una introducción de sus maestros; tal fue el caso de José Miranda, Ramón Iglesia y Silvio Zavala. Miranda, "La enseñanza de la historia en El Colegio de México", pp. 275, 277, 282. 
y mesas redondas, dar conferencias, impartir clases, escribir artículos y libros". ${ }^{18}$

Es probable que, como parte de este ejercicio académico, $\mathrm{Za}$ vala haya invitado a algunos estudiantes a participar en la revista. Con ello, lograba varios objetivos: brindar a los estudiantes material de primera mano sobre lo que se publicaba en otros países, perfeccionar la práctica de lectura, crítica y redacción, otorgar una pequeña remuneración ${ }^{19} \mathrm{y}$, por último, pero no menos importante, asegurar que la revista publicara colaboraciones de calidad.

Así, y a riesgo de pasar por alto algún nombre, ${ }^{20}$ podemos decir que este grupo estuvo conformado por 23 estudiantes (mexicanos y extranjeros) pertenecientes a las primeras generaciones del CEH, número no menor si consideramos que representa el $74 \%$ de esa población estudiantil. ${ }^{21}$ Si observamos en el apéndice la cantidad de colaboraciones por sección, se comprende que no todos se involucraron con la misma intensidad ni en el mismo

18 Luis González menciona la participación de varios estudiantes en la revista, en especial Luis Muro, quien trabajaba “a destajo” en la revista realizando numerosas reseñas. GonzÁLEz, “La pasión del nido” (100), 1976, pp. 535, 543.

19 Los colaboradores de habla hispana cobraban a razón de 3 pesos mexicanos por página. A los estadounidenses se les otorgaba más, para compensar la diferencia del tipo de cambio.

20 Para buscar las colaboraciones en la $R H A$, nos basamos en los nombres de profesores y estudiantes de la primera década del CEH que presenta Luis González, a lo que sumamos el reporte de José Miranda sobre los alumnos becarios. Véase GonzÁlez, “La pasión del nido” (100), 1976, pp. 584-597; Miranda, "La enseñanza de la historia en El Colegio de México”, p. 284. Asimismo, contrastamos esta información con la lista que generosamente nos compartió Cecilia Zuleta de estudiantes del CEH. Una vez realizado este pequeño corpus buscamos en cada número las participaciones que se plasman en el cuadro del apéndice.

21 Hay acuerdo en que fueron 31 estudiantes en el primer periodo de la década de 1940 , pero no en cuanto a sus generaciones. Moreno Toscano menciona tres [1941-1944, 1943-1946, 1946-1949. Moreno Toscano (100), 1976] mientras que los registros proporcionados por Zuleta son cuatro, pues contempla además una generación corta (1946-1947). 
tipo de trabajo. Los que entraron de lleno a la revista fueron: Ernesto de la Torre Villar, Hugo Díaz Thomé, Carlos Bosch, Alfonso García Ruiz, Julio Le Riverend, Enriqueta López Lira, Luis Felipe Muro Arias, Gonzalo Obregón, Germán Posada Mejía y Susana Uribe.

Destacaron Uribe y De la Torre. Ambos fueron incorporados oficialmente como redactores de la publicación desde 1947, pero participaron previamente en menor o mayor medida. Uribe lo hizo escribiendo reseñas desde 1942, pero aumentó su participación en 1945 (sólo participó en un número en las notas bibliográficas). En cambio, De la Torre fue más versátil al participar en más secciones. Salvo en la dedicada a los artículos, estuvo presente en todas: publicó reseñas de 1942 a 1944, varias notas bibliográficas desde 1941 y unas pocas notas necrológicas. Fundamentalmente desde 1945 coordinó la sección noticias, de la que escribió todas sus notas hasta 1949, cuando ingresan otros colaboradores a la sección. ${ }^{22}$

En cambio, si lo que se observa es el tipo de contribución en cada sección, el panorama difiere un poco. De inicio porque sólo siete estudiantes publicaron un artículo. ${ }^{23}$ En cambio, en su

22 La sección se creó en el núm. 19 (junio 1945) con la finalidad de dar a conocer las novedades institucionales de distintas bibliotecas, archivos, sociedades, academias o centros de estudio. Se nombró encargado a Ernesto de la Torre Villar, quien escribió todas las noticias hasta el núm. 25. En el siguiente (junio 1949) se introduce un cambio: las noticias van firmadas con iniciales y se incorporan otros colaboradores: Javier Malagón Barceló, Joaquín Sánchez Macgrégor, Germán Somolinos D’Ardois, Alexander Morffit, Armando Cerrato Valenzuela, Fermín Peraza Sarausa y Ernesto de la Torre Villar (quien escribe 3 de las 12 noticias). Para el siguiente número De la Torre no participa más en esta sección, aunque su nombre sigue apareciendo en las páginas legales de la publicación como miembro del equipo editorial en calidad de redactor. Cabe aclarar que el lazo con Zavala se mantuvo, al igual que con el IPGH, convirtiéndose en el secretario asistente de la Comisión de Historia de 1954 a 1965.

23 Carlos Bosch García, "Contactos diplomáticos de México con Francia, 1822-1836”, núm. 20 (dic. 1945); Ernesto Chinchilla, "Algunos aspectos de la obra de Oviedo”, núm. 28 (dic. 1949); Pablo González Casanova, "Aspectos 
calidad de practicantes, un número significativo realizó reseñas, aunque la mayoría se dedicó a colaborar en la confección de la incontable cantidad de notas bibliográficas. ${ }^{24}$ Era una labor detenida porque, además de ofrecer los datos editoriales, se opinaba de manera breve sobre el valor que tenía ese libro o artículo para la historiografía de un país o un tema. Otro espacio de colaboración fueron las notas necrológicas, pequeños textos que a manera de homenaje se realizaban sobre historiadores fallecidos. Éstas eran solicitadas casi siempre por Zavala en función del conocimiento y cercanía que existía entre el homenajeado y el autor de la nota (generalmente alumno, discípulo o al menos lector asiduo). De cualquier modo, el ser autor de una de ellas era importante para un estudiante. ${ }^{25}$

En resumidas cuentas, en esta nota hemos sintetizado a grandes rasgos aquellos trazos que señalan la pertinencia de pensar la $R H A$ como un laboratorio de prácticas, asociado a la labor formativa del CEH durante la década de 1940. El estudio de este

políticos de Palafox y Mendoza”, núm. 17 (jun. 1944); Julio Le Riverend, "Correspondencia diplomática de la delegación cubana", núm. 19 (jun. 1945); Enriqueta López Lira, "La conquista de México y su problema historiográfico", núm. 18 (dic. 1944); Germán Posada, "Singüenza y Góngora como historiador", núm. 28 (dic. 1949).

${ }^{24}$ Cabe aclarar que lo que se cuantificó en el apéndice no es la cantidad de notas bibliográficas realizadas por cada uno, sino la participación en esa sección por número. El volumen de esta sección es de más de 16000 entradas, por lo que aún no se ha podido determinar con exactitud la cantidad de notas realizadas por autor. Por esto, se registró sólo una participación por colaborador por número, dato extraído de la primera página de esta sección en cada número, donde se advierten los nombres y siglas de los colaboradores.

${ }^{25}$ Leopoldo Zea escribió sobre Antonio Caso, núm. 22 (dic. 1946); De la Torre escribió dos en el mismo número (sobre Ezequiel Chávez y Toribio Esquivel Obregón). Dos años después sobre Rómulo Velasco Cevallos, núm. 22 (dic. 1946) y núm. 25 (jun. 1948); Obregón escribió sobre Salvador Toscano, núm. 28 (dic. 1949), Posada se dedicó a su compatriota Antonio Gómez Restrepo, mientras que en el mismo número Alpuche lo hizo sobre Héctor Pérez Martínez, núm. 25 (jun. 1948); López Lira escribió una breve nota sobre Ernesto Schäfer, núm. 26 (dic. 1948). 
tema debe y puede continuarse con un análisis de cada una de estas colaboraciones para contrastarlo con otras publicaciones (otras revistas de la época o del propio Colegio de México). Finalizamos, entonces, reiterando la importancia que tiene el estudio de la revista académica para entender desde varias dimensiones lo que significó el proceso de profesionalización de la historia en México y América Latina, y algunas de las razones por las cuales el rescate de las experiencias de los editores y participantes más asiduos en la elaboración de la Revista de Historia de América tiene un notable interés para conocer antecedente esenciales de la prestigiosa revista Historia Mexicana, cuyo 70 aniversario se celebra. 
Apéndice

ESTUDIANTES DEL CEH QUE PUBLICARON EN LA

REVISTA DE HISTORIA DE AMÉRICA, 1938-1951

\begin{tabular}{|c|c|c|c|c|c|c|}
\hline \multirow[b]{2}{*}{ Colaboradores } & \multicolumn{5}{|c|}{ Secciones } & \multirow[b]{2}{*}{ Total } \\
\hline & Artículos & Reseñas & $\begin{array}{l}\text { Notas } \\
\text { biblio- } \\
\text { gráficas }\end{array}$ & Noticias & $\begin{array}{l}\text { Notas } \\
\text { necro- } \\
\text { lógicas }\end{array}$ & \\
\hline $\begin{array}{c}\text { Alpuche Sosa, } \\
\text { Helia M. }\end{array}$ & & & 1 & & 1 & 2 \\
\hline $\begin{array}{c}\text { Bosch García, } \\
\text { Carlos }\end{array}$ & 1 & 6 & 3 & 2 & & 12 \\
\hline $\begin{array}{l}\text { Carrera Stampa, } \\
\text { Manuel }\end{array}$ & & & & 1 & & 1 \\
\hline $\begin{array}{l}\text { Chinchilla A., } \\
\text { Ernesto }\end{array}$ & 1 & 1 & 3 & & & 5 \\
\hline $\begin{array}{l}\text { De la Torre V., } \\
\text { Ernesto }\end{array}$ & & & 10 & 62 & 3 & 75 \\
\hline $\begin{array}{c}\text { Díaz Thomé, } \\
\text { Hugo }\end{array}$ & & 4 & 4 & & & 8 \\
\hline $\begin{array}{l}\text { Fernández de V., } \\
\text { Manuel }\end{array}$ & & 2 & & & & 2 \\
\hline $\begin{array}{l}\text { García Ruiz, } \\
\text { Alfonso }\end{array}$ & & 8 & & 1 & & 9 \\
\hline $\begin{array}{c}\text { González C., } \\
\text { Henrique }\end{array}$ & & & 1 & 1 & & 2 \\
\hline $\begin{array}{c}\text { González C., } \\
\text { Pablo }\end{array}$ & 1 & 3 & & & & 4 \\
\hline $\begin{array}{l}\text { González y G., } \\
\text { Luis }\end{array}$ & 1 & & 2 & & & 3 \\
\hline $\begin{array}{l}\text { Gutiérrez del A., } \\
\text { Isabel }\end{array}$ & & & 2 & & & 2 \\
\hline Le Riverend, Julio & 1 & 8 & 5 & & & 14 \\
\hline $\begin{array}{l}\text { López Lira, } \\
\text { Enriqueta }\end{array}$ & 1 & 4 & 4 & & 1 & 9 \\
\hline Morales R., Sergio & & & 1 & & & 1 \\
\hline
\end{tabular}


Apéndice

ESTUDIANTES DEL CEH QUE PUBLICARON EN LA REVISTA DE HISTORIA DE AMÉRICA, 1938-1951 (concluye)

\begin{tabular}{|c|c|c|c|c|c|c|}
\hline \multirow[b]{2}{*}{ Colaboradores } & \multicolumn{5}{|c|}{ Secciones } & \multirow[b]{2}{*}{ Total } \\
\hline & Artículos & Reseñas & $\begin{array}{l}\text { Notas } \\
\text { biblio- } \\
\text { gráficas }\end{array}$ & Noticias & $\begin{array}{l}\text { Notas } \\
\text { necro- } \\
\text { lógicas }\end{array}$ & \\
\hline \multicolumn{7}{|l|}{ Muro Arias, Luis } \\
\hline Felipe & & 1 & 7 & & & 8 \\
\hline Obregón, Gonzalo & & 5 & 4 & & 1 & 10 \\
\hline Pérez M., Monelisa & & 1 & 3 & & & 4 \\
\hline $\begin{array}{c}\text { Posada Mejía, } \\
\text { Germán }\end{array}$ & 1 & 1 & 7 & & 1 & 9 \\
\hline $\begin{array}{l}\text { Sandoval, } \\
\text { Fernando }\end{array}$ & & 2 & & 3 & & 5 \\
\hline Uribe, Susana & & 12 & 4 & & & 16 \\
\hline $\begin{array}{c}\text { Velázquez, María } \\
\text { del Carmen }\end{array}$ & & 2 & 2 & & & 4 \\
\hline Zea, Leopoldo & & 2 & & & 1 & 3 \\
\hline
\end{tabular}

\section{REFERENCIAS}

Bernabéu Albert Salvador y Consuelo Naranjo Orovio, "Historia contra la 'desmemoria' y el olvido: el americanismo en el Centro de Estudios Históricos y la creación de la revista Tierra Firme (1935-1937)”, en Tierra Firme. Revista de la Sección Hispanoamericana del Centro de Estudios Históricos. Estudio Introductorio e índice, Madrid, Sociedad estatal de Conmemoraciones Culturales, Consejo Superior de Investigaciones Científicas y Publicaciones de la Residencia de Estudiantes, 2008, pp. 9-165.

Enríquez Perea, Alberto (comp.), Fronteras conquistadas. Correspondencia Alfonso Reyes/Silvio Zavala, 1937-1958, México, El Colegio de México, 1998.

Miranda, José, “La enseñanza de la historia en El Colegio de México”, en Rafael Ramírez et al., La enseñanza de la bistoria en México, México, Instituto Panamericano de Geografía e Historia, 1948, pp. 273-293. 
Moctezuma Franco, Abraham, "El camino de la historia hacia su institucionalización”, en Historia y Grafía, 25 (2005), pp. 45-78.

Mora, Carmen de, "El impulso renovador del americanismo durante la Segunda República: temas coloniales en la revista Tierra Firme”, en Revista Chilena de Literatura, 85 (2013), pp. 293-318.

PANI, Erika, "Silvio Zavala y la historia de América. Un juego de escalas", en Revista de Historia de América, 155 (jul.-dic. 2018), pp. 177-189.

Pita, Alexandra, María del Carmen Grillo y Fernando Morales, "La datificación como propuesta de análisis. El caso de la Revista de Historia de América, 1938-1948”, en Revista de Historia de América, 159 (2020), pp. 189-224.

Pita, Alexandra y María del Carmen Grillo, La Revista de Historia de América. Silvio Zavala como editor y la formación de redes académicas, 19381948 [en prensa].

Pinal Rodríguez, Karla Alejandra, Vivir para historiar, historiar para vivir. La profesionalización de la historiografía en México, una propuesta revisionista, 1850-1950, Guadalajara, Universidad de Guadalajara, 2016. 